\title{
SRH Recombination Strength of Interstitial Iron in Solar Grade Silicon
}

\author{
Mohammad Ziaur Rahman, ${ }^{*}$ Mohammad Jahangir Alam \\ Department of Electrical and Electronic Engineering, Bangladesh University of Engineering and Technology, \\ Dhaka 1000, Bangladesh
}

Received June 12, 2013; accepted August 25, 2013; published September 30, 2013

\begin{abstract}
Shockley-Read-Hall (SRH) recombination is the main determinant for an effective minority carrier lifetime in silicon. This research has focused on finding the recombination strength of interstitial iron of a low injection Shockley-Read-Hall recombination in compensated solar grade silicon by means of analytical modeling. This study has found that the strength of iron in an SRH recombination depends on the doping concentration and symmetry of a minority carrier capture cross-section. This study shows that iron becomes more recombination active at higher doping and lower symmetry of a capture cross-section.
\end{abstract}

Neutron activation analysis revealed that impurity atoms or impurity-rich particles, when dissolved in silicon or warm crystal, might form metal point defects, precipitates or microdefects [1-4]. This mechanism is probably dominant in $\mathrm{Fe}+\mathrm{Cr}+\mathrm{Ni}$-rich particles [5].

Iron (Fe) has been identified as a critical impurity concerning its electrically active incorporation in a silicon lattice. Iron precipitates at the extended defects of mc-Si form electrically active energy levels within the band gap, serving as a trap or a recombination centre, and generally being considered as unfavorable for a minority carrier lifetime [6-8].

The electronic performance of a solar cell is greatly influenced by one or more of the recombination mechanisms for a particular cell design or substrate type. When present in a silicon $\mathrm{SRH}$ recombination, $\mathrm{Fe}$ becomes a predominant mechanism for a minority carrier lifetime. The measured lifetime of a minority carrier is the primary determinant of an open circuit voltage achievable in a solar cell. The information obtained through analytical modeling of an intentionally $\mathrm{Fe}$ incorporated mc-Si solar cell will become useful in allowing further research efforts to be focused on the appropriate areas of cell design and fabrication. The parameter values for modeling are taken from the relevant published literature [4-7, 13-15].

\footnotetext{
*E-mail: ziaeee_083@yahoo.com
}

An SRH recombination lifetime depends on impurity concentration (here, Fe, for example), energy level introduced by an impurity within the band gap, capture cross section of an electron and a hole in defect energy states, and fundamental capture time constants of an electron and a hole .

Following the standard SRH lifetime equation [9-10], the injection independent $\mathrm{SRH}$ lifetime is given as

$$
\tau_{S R H, l l i}=\frac{1}{p_{0}+n_{0}}\left[\tau_{n 0}\left(p_{0}+p_{1}\right)+\tau_{p 0}\left(n_{0}+n_{1}\right)\right]
$$

Equation (1) is further simplified for p-type

$$
\tau_{S R H, l l i}=\frac{1}{p_{0}}\left[\tau_{n 0}\left(p_{0}+p_{1}\right)+\tau_{p 0} n_{1}\right]
$$

As shown in Fig 1 , in the presence of $\left[\mathrm{Fe}_{\mathrm{i}}\right]$, the n-type wafer shows to be less prone to an $\mathrm{SRH}$ recombination lifetime than its p-type counterpart. A higher lifetime for the n-type wafers is primarily due to a shorter capture period for an electron by the traps. It may also be inferred that iron in the n-type wafer has a greater influence as a trap centre rather than a recombination centre, and viceversa for the $\mathrm{p}$-type. This result suggests that solar cells made out of the n-type wafer will have higher conversion efficiency than the p-type. But other tertiary effects should be taken into account before going for a particular wafer type to make a solar cell device [11-12].

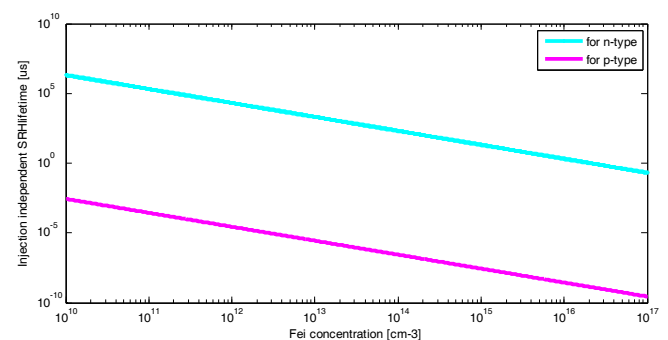

Fig. 1. Injection independent SRH lifetime of a minority carrier for varying $\left[\mathrm{Fe}_{\mathrm{i}}\right]$. 
The recombination over a deep defect state is limited by the capture of a minority carrier at a low injection level [13-14]. For a mid-gap trap, $\mathrm{n}_{1}=\mathrm{p}_{1}=\mathrm{n}_{\mathrm{i}}$ are much smaller than a doping concentration. As a result, $\tau_{S R H, l l i} \cong \tau_{n 0}$ for $\mathrm{p}$ type doping and $\tau_{S R H, l l i} \cong \tau_{p 0}$ for n-type doping [15]. This is the reason why an injection independent SRH lifetime is often called a minority carrier lifetime.

The minority carrier capture time constant is the absolute minimum of an SRH lifetime and serves as a scaling factor of the SRH lifetime. The normalization of the SRH lifetime by a minority carrier capture time constant is a direct measure of the strength of the recombination activity of a particular defect.

There are three defect energy levels, namely, deep level, shallow level and transition level, which may be responsible for an impurity present in mc-Si. The deep defect energy level lies in the middle of silicon band gap energy, the shallow level defect lies close to VB - for a hole and close to $\mathrm{CB}$ - for an electron, the transition energy level lies in the lower half of a band gap - for a hole and in the upper half - for an electron.

The normalized low injection-SRH lifetime for the p-type can be obtained from Eq. (2), such as

$$
\frac{\tau_{S R H, l l i}}{\tau_{n 0}}=\frac{1}{p_{0}}\left[p_{0}+p_{1}+\mathrm{X} n_{1}\right]
$$

where $\mathrm{X}=\frac{\tau_{p 0}}{\tau_{n 0}}$.

Figures 2, 3 and 4 show the recombination strength of Fe related defect centers in silicon. The simulated results are shown for three different energy levels (shallow, deep and transition) that might occur in the presence of an impurity in silicon.

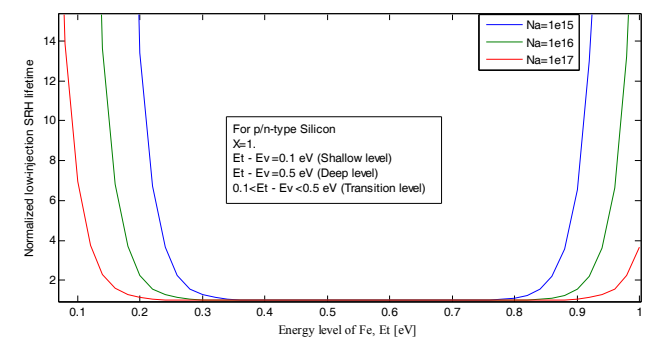

Fig. 2. Recombination strength of Fe related defect states for a symmetric capture cross- section at $300 \mathrm{~K}$.

As the symmetric capture cross section of minority carriers from Figs. 2 and 4 shows, the shallow level becomes recombination active for the higher doping concentration, the deep level exhibits maximum recombination activity over the whole doping concentration, and the recombination activity of the transition level shows strong dependence on the doping concentration for a low-injection SRH lifetime. It has been shown in Fig. 3 that for a defect energy level $E_{t}$, the smaller symmetry factor $\mathrm{X}$ is, the higher relative capture time-constant for an electron becomes, and the more effective defect for recombination is, the smaller lowinjection normalized SRH lifetime becomes.

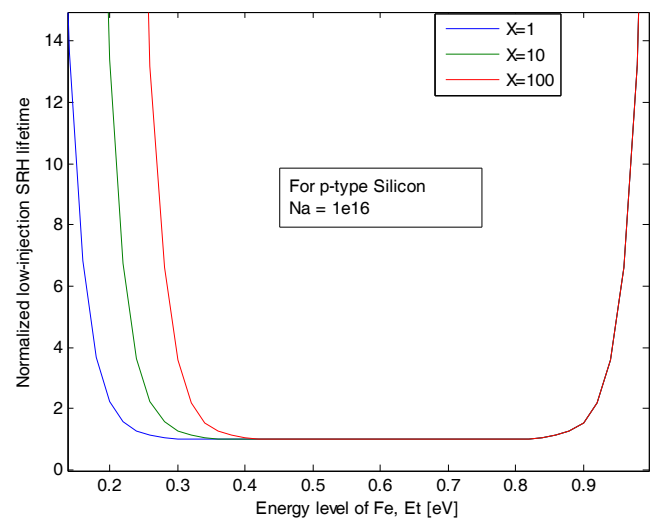

Fig. 3. Recombination strength of Fe related defect states for an asymmetric capture cross- section at $300 \mathrm{~K}$.

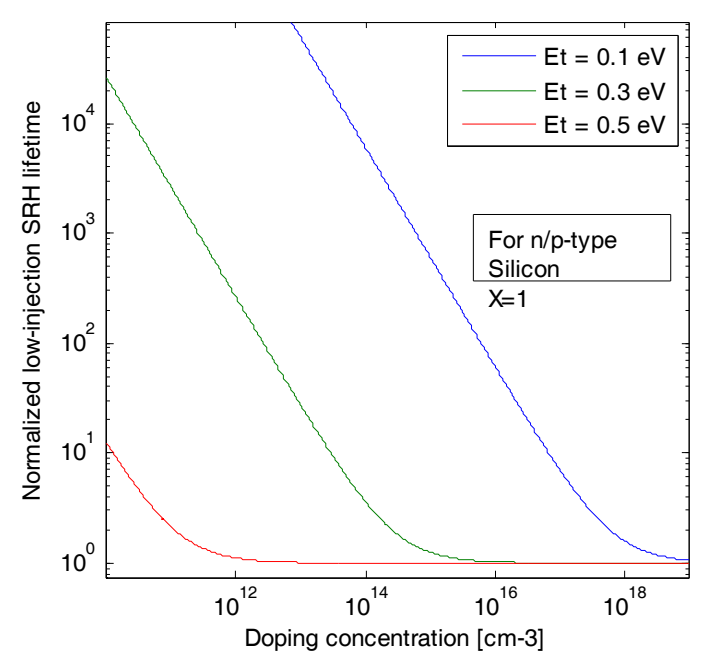

Fig. 4. Impact of doping concentration for Fe with different energy levels.

If we assume an iron related defect energy level at the upper half of the band gap, Eq. (3) reduces to

$$
\frac{\tau_{S R H, l l i}}{\tau_{n 0}}=\frac{1}{p_{0}}\left[p_{0}+\mathrm{X} n_{1}\right] \text { for } \mathrm{p} \text {-type }
$$

This study was further extended to analyze the impact of temperature [16]. To incorporate temperature dependence, $n_{l}$ and $\tau_{n 0}$ should be modified as follows 


$$
\tau_{n 0}(T)=\tau_{n 0}^{300 K}\left(\frac{T}{300 K}\right)^{-1 / 2}
$$

and

$$
n_{1}(T)=N_{C}^{300 K}\left(\frac{T}{300 K}\right)^{3 / 2} \exp \left(-\frac{E_{C}-E_{F}}{K T}\right)
$$

The overall T-dependent low-injection normalized SRH lifetime then becomes

$$
\frac{\tau_{S R H, l l i}}{\tau_{n 0}(T)}=\frac{1}{p_{0}}\left[p_{0}+\mathrm{X} n_{1}(T)\right] \text { for } \mathrm{p} \text {-type }
$$

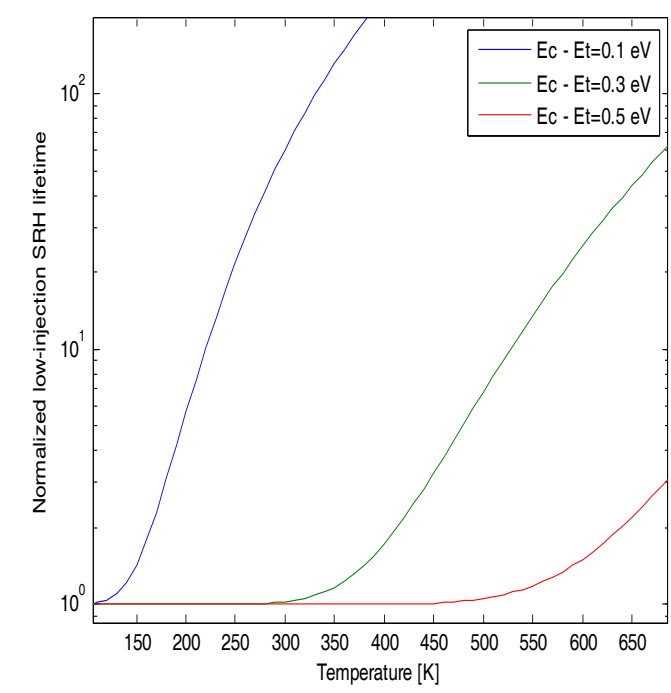

Fig. 5. Impact of Fe related defect energy levels on a T-dependent low-injection normalized SRH lifetime for a symmetric capture crosssection and a fixed doping of $10^{16} \mathrm{~cm}^{-3}$.

It can be seen from Fig. 5 that at low temperature the shallow level defect becomes less recombination active while the deep level defect becomes most recombination active. Hence, the shallow level defect shows an exponential increase in the SRH lifetime. At higher temperature, a steep increase in the SRH lifetime is observed. At higher $\mathrm{T}, \mathrm{n}_{1}(\mathrm{~T})$ in Eq. (7) dominates and the Fermi energy level approaches the mid-gap, hence a decrease in the recombination activity of a deep level defect follows.

This study demonstrated the characteristic features of recombination strength of $\mathrm{Fe}$ at varying injection and temperature levels. The presented results will help to determine defect parameters by a lifetime spectroscopy method.
Our sincere thanks go to the Department of Electrical and Electronic Engineering, BUET for providing us all the existing facilities to complete this research.

\section{References}

[1] A.A. Istratov, T. Buonassisi, R.J. McDonald, A.R. Smith, R.Schindler, J.A. Rand, J.P. Kalejs, and E.R. Weber, J. Appl. Phys. 94, 6552 (2003).

[2] T. Buonassisi, M.A. Marcus, A.A. Istratov, M. Heuer, T.F. Ciszek, B. Lai, C. Zhonghou, E.R. Weber, J. Appl. Phys. 97, 63503 (2005).

[3] T. Buonassisi, A.A. Istratov, M. Heuer, M.A. Marcus, R. Jonczyk, J. Isenberg, B. Lai, C. Zhonghou, S. Heald, W. Warta, R. Schindler, G. Willeke, E.R. Weber, J. Appl. Phys. 97, 74901 (2005).

[4] T. Buonassisi et al., Prog. Photovolt: Res. Appl. 14, 513 (2006).

[5] T. Buonassisi, A.A. Istratov, M.D. Pickett, J.P. Rakotoniaina, O. Breitenstein, M.A. Marcus, S.M. Heald, E.R. Weber, J. Crystal Growth 287, 402 (2006).

[6] A.A. Istratov, H. Hieslmair, E.R. Weber, Appl. Phys A: Material Science and Processing 69, 13 (1999).

[7] D. Macdonald, PhD thesis, Australian National University (2001).

[8] K. Graff, Metal impurities in silicon-device fabrication (SpringerVerlag, Berlin 1995).

[9] R. Hall, Phys. Rev. 87, 387 (1952).

[10] W. Shockley, W. Read, Phys. Rev. 87, 835 (1952).

[11] Y. Yoon, B. Paudyal, J. Kim, Y. Ok, P. Kulshreshtha, J. Appl. Phys. 111, 033702 (2012).

[12] J. Libal et al., J. Appl. Phys. 104, 10450 (2008).

[13] M. Ziaur Rahman, M. Jahangir Alam, Phot. Lett. Poland 5(2), 75 (2013).

[14] D.K. Schroder, American Society for Testing and Materials, ASTM STP 1340 (1998).

[15] J. Schmidt, C. Berge, A.G. Berle, Appl. Phys. Lett. 73 (15), 2167 (1998).

[16] M. Ichimura et al., J. Electrochem. Soc. 145 (9), 3265 (1998). 